\title{
ANOUILOSIS DE LA ARTICULACIÓN TEMPORO MANDIBULAR, DIAGNÓSTICO Y TERAPÉUTICA
}

\author{
ANKYLOSIS OF THE TEMPOROMANDIBULAR JOINT, DIAGNOSIS AND \\ THERAPEUTICS
}

\author{
Dra. Fátima Mazzini Torres ${ }^{1}$
}

${ }^{1}$ Doctora en Odontología. Magister en Gerencia en Servicios de Salud. Universidad Católica Santiago de Guayaquil. Ecuador. https://orcid.org/0000-0003-5321-3747

\author{
Correspondencia \\ dra.mariamazzini@hotmail.com
}

Recibido: 29-11-2021

Aceptado: 23-12-2021

Publicado: 20-01-2022

\section{RESUMEN}

Los trastornos de la articulación temporomandibular corresponden a una serie de componentes de tipo blando, óseo o combinado que pude referir o no alguna molestia en la apertura o cierre de la mandíbula; Se define a la anquilosis como la abolición completa o parcial de los movimientos de una articulación móvil. Puede ser causada por rigidez de los tejidos intra o periarticulares o por la fusión de los huesos que constituyan la articulación. Nuestro objetivo es recopilar la literatura en cuanto a clasificación, etiopatogenia, manifestaciones clínicas, diagnóstico y tratamiento de la anquilosis de la articulación temporomandibular. Metodología: Se realizó una revisión bibliográfica para desarrollar un análisis crítico reflexivo del contenido de documentos, se consideraron artículos publicados entre los años 2010 y 2020 en español. Parte de la conclusión es que la anquilosis temporomandibular es una patología incapacitante de la cavidad oral y aunque no existen datos epidemiológicos de cuanta cantidad de población la padece, la literatura sí refiere que la causa más frecuente es por traumatismo; es imprescindible para el diagnóstico una buena anamnesis y un examen clínico oportuno. Además, que entre las opciones de exámenes diagnósticos de primera elección están la resonancia magnética y la tomografía computarizada.

Palabras clave: Trastornos temporomandibulares, Anquilosis, Articulación temporomandibular.

\section{ABSTRACT}

Temporomandibular joint disorders correspond to a series of soft, bony or combined components that may or may not refer to any discomfort in the opening or closing of the jaw; Ankylosis is defined as the complete or partial abolition of the movements of a mobile joint. It can be caused by stiffness of the intra or periarticular tissues or by the fusion of the bones that make up the joint. Our objective is to collect the literature regarding classification, etiopathogenesis, clinical manifestations, diagnosis and treatment of ankylosis of the temporomandibular joint. Methodology: A bibliographic review was carried out to develop a reflective critical analysis of the content of documents, articles published between 2010 and 2020 in Spanish were considered. Part of the conclusion is that temporomandibular ankylosis is a disabling condition of the oral cavity and although there are no epidemiological data on how much of the population suffers from it, the literature does state that the most frequent cause is trauma; A good anamnesis and a timely clinical examination are essential for the diagnosis. Also, that among the diagnostic test options is magnetic resonance imaging and computed tomography.

Key Words: temporomandibular disorders, ankylosis, temporomandibular joint.

\section{INTRODUCCIÓN}

La articulación temporomandibular que forma parte de la compleja estructura craneofacial, es parte esencial del aparato masticador, que, en conjunto con las estructuras de soporte, huesos maxilares, mandibulares y demás huesos de la cara y cráneo, estructuras blandas como los músculos, vasos sanguíneos, nervios y otros como los ganglios linfáticos constituyen un componente funcional estrechamente interrelacionado entre sí y con el resto del cuerpo. 


\section{REVISTA CIENTÍFICA “ESPECIALIDADES ODONTOLÓGICAS UG”. ISSN: 2600576X ÓRGANO OFICIAL DE LA FACULTAD PILOTO DE ODONTOLOGÍA DE LA UNIVERSIDAD DE GUAYAQUIL}

Como tal, la ATM debe ser revisada como una articulación más de manera sistémica y no sólo desde alguna rama de odontología como la ortodoncia, puesto que esta articulación sinovial gínglimo-artroidal bicondílea es una de las más particulares que tiene el cuerpo humano. Es decir, que es un tipo de articulación que permite la apertura en forma de bisagra en un solo plano-es rotacional- y artoidal porque permite movimientos de traslación -deslizamiento.

Dentro de los trastornos temporomandibulares que incluyen chasquidos o crepitaciones, dolor muscular a la palpación o durante la masticación, limitación de los movimientos mandibulares, alterqciones de apertura y cierre oral. Otras sintomatologías conllevan cervicalgias, cefalea, dolor facial, otalgia, tinnitus, dolor periodontal.

Ahora bien, la anquilosis temporomandibular es un tipo de trastorno de a ATM clasificado como hipomovilidad mandibular crónica en el CIE-10. Por esta razón, la literatura coincide en determinarla como una inmovilidad anormal de la mandíbula que conlleva a problemas de apertura y cierre de la boca. Afectando así, a todas las demás funciones de la cavidad oral como son: la fonación, la masticación, la deglución y otros problemas que tienen que ver con el crecimiento facial y mandibular que puede terminar en deformidades faciales unilaterales o bilaterales como la hipoplasia mandibular o micrognatia. Además de que afecta a la higiene oral y en casos extremos la respiración. Esto sin apartarnos de que estos trastornos pueden relacionarse o dar como resultado otros tipos de trastornos como los de tipo psicológico y social

Como metodología, se realizó una revisión bibliográfica para desarrollar un análisis crítico reflexivo del contenido de documentos, se consideraron artículos publicados entre los años 2010 y 2020 en español. La búsqueda fue realizada en las bases de datos SciELO principalmente de Diciembre -Enero del 2020-2021, las palabras clave utilizadas fueron: " anquilosis, trastornos temporomandibulares, disfunciones de la ATM; se consultaron 20 artículos, de ellos fueron seleccionados 10 que cumplieron los criterios de inclusión: mención de la anquilosis temporomandibular, trastornos y disfunciones de la ATM. Los artículos que no cumplieron ese requisito fueron excluidos.

La metodología que se utilizó en este artículo, se inserta en un estudio bibliográfico, de tipo documental, de carácter descriptivo. De acuerdo a (1), "El nivel de investigación se refiere al grado de profundidad con que se aborda un objeto o fenómeno, y este puede ser exploratorio, descriptivo o explicativo" Para la realización satisfactoria de esta investigación se pretende de una manera muy suscita la definición de los requerimientos por medio de una documentación, que permiten darle soporte y mayor veracidad al artículo elaborado y adquirir nuevos conocimientos para el estudio del mismo.

La técnica utilizada fue el uso de fichas bibliográficas. En esta investigación los datos secundarios están representados por los antecedentes, tanto de las instituciones como del estudio, los artículos que han sido servido de base para la elaboración de este documento. Las categorías consideradas por los autores fueron:

- Cuáles son los trastornos de la articulación temporomandibular,

- En qué consiste la anquilosis temporomandibular.

\section{REVISIÓN DE LITERATURA}

\section{TRASTORNOS TEMPOROMANDIBULARES}

Los trastornos temporomandibulares (TTM) comprenden una serie de alteraciones intraarticulares, periarticulares, sistémicas, aunque se puede manifestar como combinaciones entre ellas (2).

Los signos y síntomas que se presentan en los TTM son numerosos, incluyen ruidos en la articulación como chasquidos o crepitación, dolor de los músculos masticadores y suprahioideos a la palpación o durante la masticación, limitación de los movimientos mandibulares, alteraciones de apertura y cierre oral, contracción involuntaria de los músculos masticadores, cefalea, dolor periodontal, dolor facial difuso, otalgia y tinnitus, así como cambios degenerativos como los observados en la artrosis y artritis reumatoide (2).

Con frecuencia la causa de la disfunción temporomandibular es una combinación de tensión muscular y problemas anatómicos dentro de las articulaciones. A veces también interviene un componente psicológico, así como otros factores (3).

- $\quad$ Síndrome de dolor miofascial: El dolor muscular y la opresión alrededor de la mandíbula es el trastorno más común que afecta a la región temporomandibular. El dolor y la limitación en el grado de abertura de la mandíbula se deben, principalmente, a la fatiga o la sobrecarga musculares, consecuencia en algunos casos de problemas de mala alineación de las arcadas dentales superior e inferior; sin embargo, con mayor frecuencia se deben a lesiones en la cabeza o en el cuello, estrés emocional, trastornos del sueño o incluso dolor de muelas (3).

- Trastorno de la articulación temporomandibular interna: En la forma más frecuente de afección articular interna de la ATM, el disco interior de la articulación está situado por delante de su posición normal. El disco se puede desplazar fuera de su sitio cuando los ligamentos que lo mantienen en su lugar se alargan o, a menudo, se estiran debido a una lesión en la articulación (esguince del ligamento).

La afección articular interna de la ATM puede producirse con reducción o sin ella

- El desplazamiento del disco acompañado de reducción articular se caracteriza por el chasquido que produce el movimiento de apertura y cierre mandibular. El 


\section{REVISTA CIENTÍFICA “ESPECIALIDADES ODONTOLÓGICAS UG”. ISSN: 2600576X ÓRGANO OFICIAL DE LA FACULTAD PILOTO DE ODONTOLOGÍA DE LA UNIVERSIDAD DE GUAYAQUIL}

disco articular se coloca en el lado opuesto a su situación habitual. Este desplazamiento sólo ocurre con la boca cerrada, cuando la boca se abre y la mandíbula se desliza hacia delante, el disco vuelve a su sitio produciendo un chasquido mientras lo hace (4).

- El desplazamiento discal sin reducir se caracteriza por una limitación en la apertura bucal al interferir el deslizamiento normal del cóndilo sobre el disco debido a la adherencia del disco, deformación, o distrofia. En esta situación, la apertura por lo general está disminuida 20-30 $\mathrm{mm}$ con una desviación de la mandíbula al lado afectado durante la apertura que generalmente se acompaña de dolor (4). La afección de la ATM puede causar inflamación alrededor de la articulación. Los trastornos internos de la ATM pueden o no ser dolorosos (3).

- Artritis: Puede aparecer artritis en la articulación temporomandibular a consecuencia de artrosis, artritis reumatoide, artritis infecciosa o un traumatismo, en particular si esta causa una hemorragia dentro de la articulación. Tales lesiones son bastante frecuentes en niños que hayan sufrido un golpe directamente en el mentón o en un lado del mentón en la articulación o a causa de la calcificación de los ligamentos que la rodean (3).

- Hipermovilidad: Se produce por el estiramiento excesivo de los ligamentos que sostienen la articulación, haciendo como si hubiera una doble articulación. En la hipermovilidad, la luxación suele producirse por la forma de las articulaciones, la pérdida de tono del ligamento (laxitud) y la tensión muscular. Puede producirse al tratar de abrir demasiado la boca, o a causa un golpe en la mandíbula (3).

\section{ANQUILOSIS DE LA ARTICULACIÓN TEMPOROMANDIBULAR}

El término "anquilosis" en griego se refiere a una soldadura u atadura, que, en el caso del complejo de la articulación temporomandibular, representa una adhesión ósea o fibrosa que conlleva a la pérdida progresiva de la función. La adhesión puede ocurrir entre la parte más superior de la apófisis condilar y la fosa glenoidea del hueso temporal o también entre cualquier tejido duro o blando del hueso mandibular y el hueso maxilar, hueso cigomático o base de cráneo (5).

La anquilosis temporomandibular es la fusión ósea, fibrosa o cartilaginosa de los componentes óseos articulares; lo que trae como resultado trastornos físicos, psicológicos y sociales como son la pérdida de la movilidad mandibular, dificultad o imposibilidad para masticar los alimentos ocasionando trastornos digestivos, aseo bucal deficiente con caries y enfermedades periodontales de complicado tratamiento dental, dificultad para hablar, y en casos extremos también para respirar, detención del crecimiento y desarrollo mandibular que puede ser uni o bilateral y que deriva en deformidades faciales como hipoplasia mandibular o micrognatia (6).
La literatura define a la anquilosis como una inmovilidad anormal de la mandíbula, desorden que lleva a una restricción de la apertura bucal con reducción parcial de los movimientos mandibulares o una completa inmovilidad de la mandíbula (7).

Es una de las más serias e incapacitantes condiciones patológicas que pueden ocurrir en la región maxilofacial ya que interfiere en su funcionalidad pudiendo afectar el habla, la masticación, la higiene oral y en el crecimiento facial y mandibular (7).

La anquilosis de la articulación temporomandibular (ATM) puede presentarse como una afección congénita casi siempre asociada a un síndrome de malformación fetal. En la adolescencia la consecuente desarmonía dentomaxilofacial se manifiesta con graves problemas en la oclusión dentaria por sobrecrecimento del maxilar, extrusión y protrusión de los dientes incisivos superiores que, con la mandíbula hipotrófica, pueden determinarles un perfil de pájaro, con el tercio medio de la cara desproporcionado en relación con el tercio inferior, acompañado de alteración asimétrica de la cara a lo que puede asociarse una cierta dificultad respiratoria al dormir (8).

\section{Etiopatogenia}

La anquilosis de la articulación temporomandibular (ATM) a menudo se produce por un traumatismo o una infección, pero puede ser congénita o deberse a una artritis reumatoide. Hay una limitación indolora y crónica del movimiento. Cuando la anquilosis produce una interrupción del crecimiento condilar, aparece una asimetría facial, si el tejido cicatricial se daña por exigencia funcional, aparecerán síntomas de artritis inflamatoria aguda (9).

Es más frecuente que se asocie con traumatismo (13\% $100 \%)$, infección local o sistémica (10\% -49\%), o enfermedad sistémica como la espondilitis anquilosante, la artritis reumatoide y la psoriasis (10\%). Puede también ser secundario a la cirugía de la ATM (10).

En las infecciones tienen un origen dental, del oído medio o del proceso mastoidal. La hipótesis de que el hematoma intraarticular solo puede conducir a la anquilosis de la ATM se basa en su organización y posterior osificación (10).

La radioterapia, la extirpación quirúrgica de tumores de la ATM también puede provocar hipomovilidad mandibular. La radioterapia produce fibrosis, cicatrización e induración de los tejidos blandos que rodean las ATM. Esta en última instancia, puede dar lugar a anquilosis fibrosa intraarticular (10).

Si la anquilosis de la ATM se produce durante la infancia, esta condición que involucra la fusión ósea del hueso mandibular y temporal, causa deformidades dento faciales importantes en el crecimiento y desarrollo del niño y el adolescente; algunas de las características más comunes en estos pacientes- que se encuentran en edades comprendidas entre los 8 y 16 años son: asimetría facial, retrusión mandibular, limitación de la apertura bucal, pérdida de la dimensión vertical, 


\section{REVISTA CIENTÍFICA “ESPECIALIDADES ODONTOLÓGICAS UG”. ISSN: 2600576X ÓRGANO OFICIAL DE LA FACULTAD PILOTO DE ODONTOLOGÍA DE LA UNIVERSIDAD DE GUAYAQUIL}

desplazamiento mandibular hacia el lado afectado, secuelas de traumatismos intra o extraorales en la zona sinfisiaria, y en casos avanzados además: micrognatia, compromiso de la vía aérea cursando con infecciones repetitivas del tracto respiratorio superior inclusive asociándose con un síndrome complejo de apnea obstructiva del sueño (5).

Cuando la anquilosis se da en niños la parte emocional es muy importante, los padres es un factor importante para obtener resultados favorables. Sin embargo, cuando la anquilosis ocurre en adultos, generalmente solo está comprometido la apertura de la mandíbula que se encuentra restringida (10).

\section{- $\quad$ Inervación del ATM}

Está inervada por el nervio trigémino que provee inervación sensorial y motora a los músculos que la controla. Ramas del nervio mandibular proveen la inervación aferente. La mayor parte está dada por el nervio auriculotemporal. La temporal profunda y el nervio maseterino provee inervación adicional. (11).

\section{- Vascularización del ATM}

Los vasos predominantes son: arteria temporal profunda desde la zona posterior, meníngea media desde la zona anterior y la arteria maxilar interna desde la zona inferior. Otras arterias: auricular profunda, timpánica anterior, faríngea ascendente; el cóndilo recibe irrigación a través del hueso esponjoso y de los vasos nutricios. (11).

\section{- Clasificación de las anquilosis de la articulación temporomandibular}

Puede ser clasificada de distintas maneras. Dentro de las cuales encontramos la clasificación de acuerdo con la localización de ocurrencia, clasificación según el tipo de tejido involucrado, según su extensión, según sus grados de severidad y según su origen. (7).

- $\quad$ Según su localización

- Intracapsular: La patología involucra estructuras intracapsulares.

- Extracapsular: La patología involucra estructuras extracapsulares, pudiendo involucrar además estructuras intracapsulares (7).

- Según el tipo de tejido involucrado

- Ósea: Afectación de tejidos óseos articulares.

- Fibrosa: Afectación de tejidos fibrosos articulares.

- Fibroósea: Combinación entre afectación de tejidos óseos y fibrosos articulares (7).

- Según su extensión

- Completa: Fusión de las estructuras de forma completa.

- Incompleta: Fusión de las estructuras de forma incompleta (7).

- $\quad$ Según el origen

- Verdadera: cuando el daño es causado intraarticularmente por la adhesión del cóndilo mandibular a la cavidad glenoidea (5).
- Falsa o pseudo-anquilosis: cuando las estructuras extraarticulares son la causa de la hipomovilidad mandibular, como son la hiperplasia de la apófisis coronoides, fracturas no reducidas del arco cigomático, fibrosis post-radiación del músculo temporal y desórdenes neurológicos, entre otros (5).

- Según su severidad

- Tipo 1: Cabeza del cóndilo se encuentra presente pero deformada.

- Tipo 2: La fusión se produce entre la cabeza del cóndilo y la superficie articular.

○ Tipo 3: Formación de un bloque óseo entre la rama de la mandíbula y el hueso cigomático.

○ Tipo 4: La anatomía de la ATM se encuentra totalmente alterada (7).

\section{- Manifestaciones clínicas}

Entre las manifestaciones clínicas se plantea que algunos pacientes pueden ser asintomático, pero por lo general se encuentran dolor a la masticación, ruidos o chasquidos, dificultades en el movimiento de la articulación que puede favorecer la aparición de disturbios nutricionales, como consecuencia, problemas en el crecimiento y desarrollo. Se describen inconvenientes para conservar la higiene, que puede favorecer los procesos infecciosos de la cavidad bucal y respiratoria, además de la pérdida de dientes; dificultades en el habla, respiratorias y deformidades o desfiguración facial que puede ocasionar daño psicológico a la persona poseedora de este problema de salud (12).

\section{- Diagnóstico}

La valoración integral del complejo articular debe incluir la valoración de los tejidos orofaciales, la función muscular y neurológica, el estudio de la oclusión, los movimientos mandibulares y la identificación de los posibles hábitos parafuncionales (7).

Si bien todo lo anterior es imprescindible en muchas ocasiones debemos apoyarnos en las diferentes técnicas de diagnóstico por imagen para poder etiquetar el cuadro clínico que aqueja al paciente. Radiográficamente la anquilosis demuestra aspectos bien característicos que facilitan el diagnostico, sin embargo, su análisis en las técnicas radiográficas convencionales, en la mayoría de la vez no se presenta de forma clara (7).

De las diferentes técnicas radiográficas que nos ayudan al diagnóstico: proyección panorámica, proyección transfaríngea (infracraneal), proyección transcraneal lateral, proyección transmaxilar anteroposterior y otras; son la proyección panorámica y la transcraneal lateral las que todavía, y con muchas reservas, en la actualidad podrían tener alguna indicación dentro de la patología de la ATM. Hoy en día teniendo en cuenta que hay pruebas más resolutivas, no está justificado el coste radiológico que representan, teniendo en cuenta, además, que la anquilosis del tipo fibroso no es visible en las imágenes radiográficas convencionales (7). 


\section{REVISTA CIENTÍFICA “ESPECIALIDADES ODONTOLÓGICAS UG”. ISSN: 2600576X ÓRGANO OFICIAL DE LA FACULTAD PILOTO DE ODONTOLOGÍA DE LA UNIVERSIDAD DE GUAYAQUIL}

Otras técnicas, como la tomografía computarizada (TC), provee imágenes con importante información en lo que concierne a las características individuales de lesiones patológicas, información que es particularmente útil para diagnósticos y tratamientos. La TC presenta muchas ventajas, pues complementa las informaciones relacionadas con la extensión y límite de la zona de anquilosis (7).

La tomografía computarizada de haz cónico (CBCT) sobresale como una alternativa a la TC, convirtiéndose en una relevante herramienta para el diagnóstico imagenológico óseo de la zona oral y maxilofacial. Además, el CBCT permite obtener imágenes usando una dosis más baja de radiación, tiempo más corto de exanimación al paciente, y costos más bajos que las $\mathrm{TC}$, lo que hace de su rutina viable para procedimientos orales y maxilofaciales (7).

Por último, encontramos la Resonancia Nuclear Magnética (RNM), que es la técnica de elección para el diagnóstico funcional y patológico de la ATM, no sólo por la información anatómica y funcional que aporta sino porque es una técnica que evita las radiaciones ionizantes. Es, además, la prueba de referencia para identificar posiciones del disco articular. La RNM es capaz de establecer la presencia de anquilosis ósea, fibrosa y fibroósea, siendo determinante en el diagnóstico de estas dos últimas (7).

\section{- $\quad$ Tratamiento}

El tratamiento de la anquilosis temporomandibular ha sido bien documentado y reportado en múltiples artículos y libros referentes a esta patología, estableciéndose protocolos encaminados a restablecer la movilidad mandibular y restaurar la anatomía articular, a la vez de favorecer el desarrollo y crecimiento de las estructuras óseas dañadas (6).

El tratamiento de la anquilosis de ATM ha sido discutido en la literatura por más de 200 años, no existiendo consenso en la literatura existente sobre cuál es el mejor. Por lo tanto, la evaluación clínica previa y la técnica quirúrgica utilizada son importantes. Aunque indudablemente, el tratamiento debe siempre proporcionar y mantener una apertura bucal postoperatoria satisfactoria y una correcta funcionalidad de la mandíbula al largo plazo para prevenir una recidiva del cuadro (7).

Dentro de los principios en el tratamiento de la anquilosis de ATM, encontramos una cirugía lo más precoz posible una vez establecido el diagnóstico, preservación de la altura de la rama mandibular, prevención de recurrencias con material de interposición y terapia física postoperatoria. Así, el objetivo del manejo sería buscar la extirpación del bloque anquilótico obteniendo una correcta morfología; devolver la función articular; y corregir una posible deformidad dentofacial y/o alteración del crecimiento (7).

Las técnicas quirúrgicas en el tratamiento de la anquilosis de ATM son diversas. Algunos investigadores plantean que para lograr el éxito es imprescindible la interposición de algún material en el espacio creado, siendo la importancia de esto prevenir la recurrencia de la patología; otros, en cambio, han demostrado que crear y mantener el espacio es lo más importante.

Un tercer grupo opina que los resultados son iguales tanto si se utiliza o no un material interpuesto en dicho espacio. Este material interpuesto puede ser de tipo autógeno o aloplástico, entre los materiales autógenos tenemos la ventaja de no haber rechazo por parte del paciente, pero en contrapartida, tenemos la desventaja de la morbilidad quirúrgica que se presenta.

Los materiales aloplásticos no debilitan al paciente, pues no hay área donadora, pero pueden causar el rechazo del organismo al material de elección. Algunos de los materiales aloplásticos poseen la desventaja del alto costo y dificultad de confección de la pieza para substitución de la anquilosis como el oro y el acero inoxidable. Otros tienen bajo costo y facilidad de trabajo, como es el caso de la resina acrílica y del papel celofán (7).

\section{CONCLUSIONES}

La anquilosis temporomandibular es uno de los trastornos de la ATM que tiene que ver con la fusión del cóndilo de la mandíbula con la porción petrosa del hueso temporal. Es una patología incapacitante de la cavidad oral y aunque no existen datos epidemiológicos de cuanta cantidad de población la padece, la literatura sí refiere que la causa más frecuente es por traumatismo.

Es imprescindible para el diagnóstico una buena anamnesis y un examen clínico oportuno. Además, que entre las opciones de exámenes diagnósticos de primera elección están la resonancia magnética y la tomografía computarizada. Esto nos exhorta como operadores a derivar con el especialista capacitado para dar un buen resultado en el tratamiento.

Entre las opciones de tratamiento que son muy diversas y discutidas entre profesionales, puesto que, va a depender de factores como la edad del paciente y la causa, lo que va a determinar el abordaje quirúrgico. Aunque en lo que sí coinciden es que el abordaje quirúrgico debe cumplir con un mismo fin: evitar la recurrencia y recuperar la máxima funcionalidad.

Existe un consenso entre autores en cuanto a que el diagnóstico temprano es vital para establecer un pronóstico y éxito del tratamiento. Esto también se acompaña de que en ciertos casos se suman cuidados especiales y rehabilitación postoperatoria.

Una complicación frecuente en los tratamientos para los casos que se necesita injerto es alguna infección o que el organismo no reaccione bien al injerto. Aunque, se han reportado casos de fracaso en el tratamiento por pobre vascularización, en realidad no es una causa frecuente.

Entre las técnicas de abordaje se encuentran la utilización de instrumentos ultrasónicos para evitar el daño de las 


\section{REVISTA CIENTÍFICA “ESPECIALIDADES ODONTOLÓGICAS UG”. ISSN: 2600576X ÓRGANO OFICIAL DE LA FACULTAD PILOTO DE ODONTOLOGÍA DE LA UNIVERSIDAD DE GUAYAQUIL}

estructuras que circundan la ATM, tales como: venas, arterias y nervios, aparte de los componentes musculares de la zona.

Una de las complicaciones de la anquilosis es que debido a la masa ósea que se está formando no permite reconocer las estructuras aledañas debido al crecimiento óseo y fibroso, entonces estas venas, arterias o nervios pueden estar muy cerca de la zona que se va a tratar quirúrgicamente. Además que puede cerrar forámenes por donde pasan estructuras íntimamente relacionadas como la carótida interna.

\section{REFERENCIAS BIBLIOGRÁFICAS}

1. Arias, Fidias. El proyecto de investigación. Introducción a la metodología científica. Caracas : Episteme, 2012. 6ta.

2. Trastornos temporomandibulares. Lescas Méndez, Octavio, y otros. 1, 2012, Vol. 55.

3. Klasser, Gary. Manual MSD. Trastornos temporomandibulares. [En línea] julio de 2020. [Citado el: 8 de enero de 2021.] https://www.msdmanuals.com/esec/hogar/trastornos-bucales-y-dentales/trastornostemporomandibulares/trastornos-temporomandibulares.

4. Trastornos de la asrticulación témporo-mandibular. Aragón, M C, Aragón , F y Torres , L M. 7, 2010, Vol. 12.

5. Manejo contemporáneo de la Anquilosis de la Articulación Temporomandibular en el niño y el adolescente: Reporte de Casos. Broggi Ángulo, Óscar Alfredo, y otros. 2, Lima : Acta médica peruana, 2013, Vol. 30.

6. Tratamiento de anquilosis temporomandibular con materiales aloplásticos en niños (reporte de 3 casos). Montañez, Francisco Mercado. 1, Torreón : Revista ADM, 2018, Vol. 75.

7. Anquilosis de la articulación temporomandibular: Una revisión de la literatura. Molina, y otros. 5, Chile : s.n., 2013, Vol. 29.

8. Tratamiento simultáneo de anquilosis temporomandibular unilateral congénita y del microlaterognatismo asociado. Varela, José Felipe Basulto, y otros. 1, La Habana : Rev Cubana Estomatol, 2013, Vol. 50.

9. Mehta, Noshir. Manual MSD. Anquilosis de la articulación temporomandibular Joint (ATM). [En línea] febrero de 2019. [Citado el: 10 de enero de 2021.] https://www.msdmanuals.com/es/professional/trastornosodontol\%C3\%B3gicos/trastornos-

temporomandibulares/anquilosis-de-la-articulaci\%C3\%B3ntemporomandibular-joint-atm.

10. Deza Palle, Jonathan William. Tratamiento quirúrgico de la anquilosis de la articulación temporomandibular. Lima:
Universidad Nacional Mayor de San Marcos, Facultad de Odontología, Unidad de Posgrado, 2019.

11. Anquilosis temporomandibular. Liberación, reconstrucción con injerto de apófisis coronoides y cartílago auricular: Presentación de caso clínico. Ravelo, José, Lopez, Juan Carlos y Ruiz, Rafael. 2, Ciudad de México: Revista Odontológica Mexicana, 2011, Vol. 15. 2.

12. Anquilosis de la articulación temporomandibular. Vite Vera, Erika Fabiola, y otros. 3, Cuba : La Habana, 2019, Vol. 21.

\section{Conflictos de intereses}

Los autores señalan que no existe conflicto de intereses durante la realización del estudio, no se recibió fondos para la realización del mismo, el presente solo fue sometido a la Revista Científica

"Especialidades odontológicas UG" para su revisión y publicación

\section{Financiamiento}

Los autores indican la utilización de fondos propios para la elaboración del trabajo de investigación.

\section{Declaración de contribución}

Todos los autores han contribuido en elaboración del trabajo de investigación, en las diferentes partes del mismo. 